\section{Dermatitis und Kontaktallergie: Systematisch falsch untersucht?}

\begin{abstract}
Studien, in denen nach einer Assoziation der atopischen Dermatitis mit Kontaktallergien gesucht wurde, verliefen in der Vergangenheit oft negativ. War dafür ein systematischer Fehler verantwortlich?
\end{abstract}

$\mathrm{D}$ ie Idee, das Fehlen einer Verbindung von atopischer Dermatitis (AD) und einer Kontaktsensibilisierung gehe womöglich auf eine Schieflage des Datenmaterials zurück, beruht auf der „BypassHypothese“. Danach werden die Ergebnisse verzerrt, weil mit Nickel und Thiomersal zwei Hauptallergene nicht nur transdermal, sondern auch durch Piercing und Injektionen direkt in die Haut gelangen. Die Filaggrin-Barriere wird dabei mechanisch überwunden. Die Empfindlichkeit auf diese Stoffe ist bei den betroffenen Personen erhöht - unabhängig von Strukturveränderungen der Haut, wie sie für die $\mathrm{AD}$ typisch sind.

Allergologen um Jacob Thyssen vom dänischen Nationalen Allergieforschungszentrum in Kopenhagen haben dies nun überprüft. Sie unterzogen 3.202 Erwachsene im Alter von 18 bis 69 Jahren einem Patchtest, untersuchten deren FilaggrinGenotyp und fragten nach, ob bei den
Probanden eine $\mathrm{AD}$ diagnostiziert worden war.

Wurden die Substanzen Nickel und Thiomersal aus den Analysen ausgeklammert, ergab sich ein signifikanter Zusammenhang von $\mathrm{AD}$ mit dem Bestehen einer Kontaktsensibilisierung: Personen, die eine Kontaktsensibilisierung aufwiesen, hatten 2,5-mal häufiger eine $\mathrm{AD}$ als Studienteilnehmer, die auf keinen der getesteten Stoffe reagierten. Zog man Nickelsensibilisierungen mit in die Berechnung ein, war diese Assoziation hingegen nicht mehr nachzuweisen. Filaggrinmutationen spielten dabei übrigens keine Rolle. Untermauert wurden die Befunde auch durch eine Subgruppenanalyse: Bei Frauen ohne Piercing korrelierte auch die Nickelsensibilisierung positiv mit einer $\mathrm{AD}$.

Fazit: Die Studie untermauert die BypassHypothese. Bei Substanzen wie Nickel, die etwa durch ein Piercing direkt mit tieferen

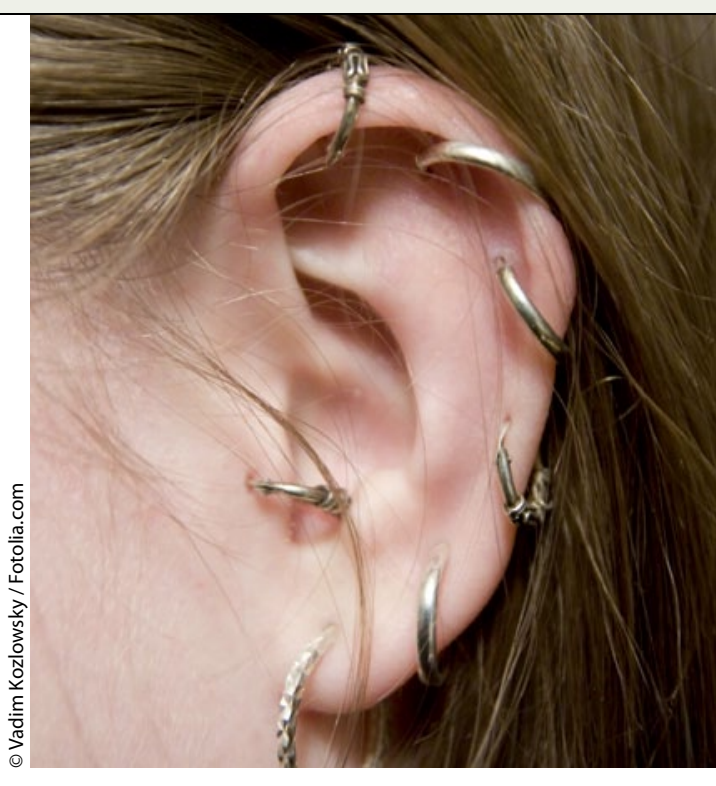

Beim Durchstechen der Ohrläppchen wird die Hautbarriere auch bei Personen überwunden, die keine atopische Dermatitis aufweisen. Mögliche Folgen sind Kontaktsensibilisierungen gegen Nickel und eine verzerrte Statistik.

Hautschichten in Kontakt kommen, sind Kontaktallergien nicht mehr zwingend mit strukturellen Hautveränderungen einer $\mathrm{AD}$ assoziiert. Dr. Robert Bublak

Thyssen JP et al. Contact sensitization to common haptens is associated with atopic dermatitis: new insight. Br J Dermatol 2012; 166: 1255-61

\title{
Selbstverletzungen bei Kindern
}

Phänomene wie Schneiden und Ritzen der Haut, aber auch andere nicht suizidale Selbstverletzungen (NSSV) können sich schon früh in der Kindheit zeigen. Eine US-amerikanische Studie ergab dabei deutliche Alters- und Geschlechtsunterschiede.

elbstverletzungen sind keine neu$S$ artige Erscheinung. Erste Berichte stammen aus dem fünften Jahrhundert vor Christus und sind in verschiedenen Glaubensrichtungen auch als religiös motivierte Handlungen bekannt geworden. Um herauszufinden, wie häufig NSSV aktuell bereits von Kindern praktiziert werden und welche Methoden sie dabei einsetzen, befragten Forscher 665 amerikanische Kinder und Jugendliche zwischen sieben und 16 Jahren.

Insgesamt $8 \%$ der Kinder und Jugendlichen berichteten über eigene Erfahrungen mit NSSV. Etwa bei jedem Dritten handelte es sich um ein einmaliges Ereig- nis. Das Spektrum der Verletzungen reichte vom Schneiden und Ritzen sowie Verbrennen der Haut über das Einritzen und Einstechen von Mustern und Selbsttätowierungen bis hin zur Selbstgeißelung. Aber die Kinder bissen sich auch, zogen sich schmerzhaft an den Haaren, rannten gegen Wände und warfen sich auf scharfe Gegenstände.

Während das Schlagen insgesamt eher eine Methode der Jungen war (55\% vs. $42 \%)$, hantierten die Mädchen auffallend häufig mit Messern (64\% vs. 15\%). Mit zunehmendem Alter traten die Geschlechtsunterschiede immer deutlicher hervor. In der dritten Klasse brachten sich Jungen et- was häufiger als Mädchen selbst Verletzungen bei ( $8,5 \%$ vs. $6,8 \%)$ - bei Jugendlichen in der neunten Klasse dagegen hatten sich die Quoten eindeutig ins Gegenteil verkehrt (19\% Mädchen vs. 5\% Jungen).

Fazit: Diese erste Studie mit auch jüngeren Kindern hat gezeigt, dass Selbstverletzungen nicht allein ein Problem der Jugendlichen ist. Zudem wurden Geschlechtsunterschiede deutlich. Die Daten der amerikanischen Studie sind vergleichbar mit der europäischen CASE-Studie aus dem Jahr 2008. Diese ergab, dass sich in Europa 4-10\% der 15- bis 16-Jährigen Verletzungen beibringen. Auch hier waren weibliche Jugendliche häufiger betroffen als männliche. Dr.Christine Starostzik

Barrocas AL et al. Rates of nonsuicidal selfinjury in youth: age, sex, and behavioral methods in a community sample. Pediatrics 2012 June 11 [Epub ahead of print] 